\title{
Evaluación en Laboratorio de la Patogenicidad de Aislamientos Nativos de Beauveria sp. y Metarhizium anisopliae para el Control de Premnotrypes vorax
}

\author{
Enrique Barriga, Pablo Landázuri, Patricio \\ Gallegos ${ }^{1}$, Roger Williams ${ }^{2}$
}

\begin{abstract}
Resumen
El presente estudio determinó la patogenicidad de aislamientos nativos de Beauveria sp. y Metarhizium anisopliae recolectados en localidades de Carchi, Chimborazo, Cotopaxi y Pichincha (Ecuador), sobre Premnotrypes vorax (Hustache) adultos.
\end{abstract}

Se evaluaron 19 tratamientos en un DCA con 5 observaciones. La unidad experimental fue 20 insectos. Las localidades se constituyeron en los tratamientos: $\mathrm{t} 1=\mathrm{SJ}$. Minas; $12=$ Santa Catalina; $\mathrm{t} 3=$ Chanchaló; $\mathrm{t} 4=$ Sablog; $\mathrm{t} 5=$ Huacona SJ.; $16=\mathrm{SM}$. de Cuba; $\mathrm{t}=$ FCA CADET; t8= Guabo, t9=S.J. Huaca, t10=Tumbaco, t11=Cabañal, $\mathrm{t} 12=\mathrm{Pull}$ Chico, $\mathrm{t} 13=$ Trebon, $\mathrm{t} 14=4$ Esquinas, $\mathrm{t} 15=$ Compostera, t16=FCA ESPOCH, t17=Yacubamba, t18=San Francisco y t0= testigo. Las variables analizadas fueron 1) Mortalidad de insectos a los 5,10,15, 20, y 25 días la infección, 2) Tiempo letal medio TL 50.

Los aislamientos se multiplicaron en PDA (más harina de $P$. vorax) en cajas petri, Posteriormente los insectos se infectaron en una solución de esporas por dos minutos, para luego colocarlos en cajas plásticas con tierra esterilizada.

Los mejores aislamientos para las dos variables fueron: $\mathrm{t} 3, \mathrm{t} 5$, $\mathrm{t} 9$ y 117 ; el $100 \%$ de mortalidad de adultos de $P$. vorax alcanzaron a los 10 días de infección y el tiempo letal medio TL 50 alcanzaron a los 6.6, 6.4, 6,6 y 6.3 días, respectivamente. Los aislamientos en los que el insecto presentó menor susceptibilidad fueron t2, t7, t15 y t16, estos necesitaron más tiempo para alcanzar el 100\% de mortalidad de adultos de $P$. vorax y el tiempo letal medio también fue superior. El testigo no registro mortalidad.

Palabras claves adicionales: control biológico, bioinsecticidas

1. INIAP. E-mail:gallegos@fpapa. org. ec. ;Casilla 340 INIAP Quito. Ecuador.

2. Proyecto IPM-CRSP Ohio State University 1680 Madison Av. Wooster, Ohio 44691, correo electrónico: williams14@osu.edu 


\title{
Pathogenicity Evaluation of Native Beauveria sp. and Metarhizium anisopliae to Control Premnotrypes vorax
}

\begin{abstract}
Summary
In laboratory assays, pathogenicity of native Beauveria sp. and Metarrhizium anisopliae was tested. Both organisms were collected in Carchi, Chimborazo, Cotopaxi, and Pichincha (Ecuador) growing on Premnotrypes vorax (Hustache) adults. Localities were considered as treatments: $\mathrm{t} 1=\mathrm{SJ}$. Minas, 12= Santa Catalina, $\mathrm{t} 3=$ Cháncalo, $\mathrm{t} 4=$ Sablog, $\mathrm{t} 5=$ Huacona SJ., $\mathrm{t} 6=$ $\mathrm{SM}$. de Cuba, $\mathrm{t} 7=\mathrm{FCA}$ CADET, $\mathrm{t} 8=\mathrm{Guabo}, \mathrm{t} 9=\mathrm{S} . \mathrm{J}$. Huaca, $110=$ Tumbaco, t11 = Cabañal, t12 = Pulí Chico, t13= Trebon, t14= 4 Esquinas, $\mathrm{t} 15=$ Compostera, $\mathrm{t} 16=\mathrm{FCA} E S P O C H, \quad t 17=$ Yacubamba, $118=$ San Francisco, and $\mathrm{t} 0=$ Check Treatment. Analyzed variables were: 1) Insect mortality at 5, 10, 15, 20, and 25 days after infection, 2) Mean lethal time LT50. Fungi isolates were multiplied in PDA (plus $P$. vorax meal) in Petri dishes. Infection procedure was to introduce the insects in a spore suspension for two minutes and to place them in plastic boxes containing sterile soil. The best treatments for the two variables were: $13, \mathrm{t} 5, \mathrm{t} 9$, and $\mathrm{t} 17$. The $100 \%$ mortality of $P$. vorax adults was after 10 days from infection, and the TL50 was reached after $6.6,6.4,6.6$, and 6.3, respectively. Treatments that showed less susceptibility to inoculations were: $\mathrm{t} 2, \mathrm{t} 7, \mathrm{t} 15$, and $\mathrm{t} 16, P$. vorax needed a longer time to reach $100 \%$ adult mortality, and TL50 was also longer. The control treatment did not show mortality.
\end{abstract}

Additional Índex words:

Biological control, bioinsecticides

\section{Introducción}

El primer paso para el desarrollo de un programa de control biológico exitoso, mediante entomopatógenos, es la recolección de aislamientos nativos y la determinación de su patogenicidad. El presente trabajo evaluó la patogenicidad de los hongos Beauveria sp. y Metarhizium anisopliae recolectados de insectos adultos de $P$. vorax

La recolección, previa, de los hongos indicados se realizó en diferentes áreas de producción de papa del Ecuador(Carchi, Chimborazo Cotopaxi y Pichincha). Estos hongos provienen de adultos de $P$. vorax infectados naturalmente en el campo y capturados mediante trampas según la metodología descrita por Gallegos et al. (3). 
La patogenicidad se evaluó en términos de mortalidad y de tiempo letal medio (TL 50). El objetivo planteado fue evaluar en laboratorio la patogenicidad de aislamientos nativos de Beauveria sp. y Metarhizium anisopliae en el adulto del "Gusano blanco" de la papa, Premnotrypes vorax (Hustache).

\section{Materiales y Métodos}

\section{Materiales}

- Aislamientos de hongos entomopatógenos (13 aislamientos de Beauveria sp. y 5 de $M$. anisopliae procedentes de cepario del Departamento de Protección Vegetal de la Est. Esp. Sta Catalina -INIAP).

- Insectos adultos de "Gusano Blanco" (P. vorax )

- Material de laboratorio para hongos entomopatógenos

\section{Tratamientos}

\begin{tabular}{|c|c|c|c|c|}
\hline & \multirow[b]{2}{*}{ Aislamientos } & \multicolumn{3}{|c|}{ Origen } \\
\hline & & Provincia & Cantón & Localidad \\
\hline t1 & Beauveria sp. & Pichincha & Quito & San José de Minas \\
\hline $\mathrm{t} 2$ & Beauveria sp. & Pichincha & Quito & E.E. Santa Catalina \\
\hline t3 & Beauveria sp. & Cotopaxi & Salcedo & Chanchalo \\
\hline t4 & Beauveria sp. & Chimborazo & Guarnote & Sablog \\
\hline $\mathrm{t} 5$ & Beauveria sp. & Chimborazo & Guarnote & Huacona \\
\hline t6 & Metarhizium anisopliae & Carchi & Tulcán & Santa Martha de Cuba \\
\hline t7 & Metarhizium anisopliae & Pichincha & Mejía & E.E. Santa Catalina \\
\hline t8 & Metarhizium anisopliae & Pichincha & Quito & U. Central F. Agronomomía \\
\hline t9 & Beauveria sp. & Carchi & Tulcán & Santa José de Huaca \\
\hline t10 & Beauveria sp. & Pichincha & Quito & Tumbaco \\
\hline $\mathrm{t} 11$ & Beauveria sp. & Cotopaxi & Latacunqa & Cabañal \\
\hline $\mathrm{t} 12$ & Beauveria sp. & Chimborazo & Guano & Pulí Chico \\
\hline t13 & Beauveria sp. & Chimborazo & Guano & Esquina (Trebon) \\
\hline t14 & Beauveria sp. & Chimborazo & Guano & 4 Esquinas \\
\hline t15 & Metarhizium anisopliae & Pichincha & Mejía & Sta. Catalina (Compostera) \\
\hline $\mathrm{t} 16$ & Metarhizium anisopliae & Chimborazo & Riobamba & $\begin{array}{l}\text { Escuela Politécnica F. } \\
\text { Agronomía. }\end{array}$ \\
\hline $\mathrm{t} 17$ & Beauveria sp. & Cotopoxi & Pujilí & Yacubamba \\
\hline t18 & Beauveria sp. & Carchi & El Ángel & San Francisco \\
\hline t0 & Testigo absoluto & & & \\
\hline
\end{tabular}

\section{Análisis estadístico}

\section{Diseño experimental}

Se utilizó un DCA con19 tratamientos y 5 observaciones. 


\section{Prueba de significación}

Se utilizó la prueba de Tukey al 5\% para tratamientos y DMS AL 5\% para las comparaciones ortogonales

\section{Regresión}

Para el cálculo del Tiempo Letal Medio o TL 50, se estableció una regresión entre el porcentaje de mortalidad de cada tratamiento vs los días de evaluación.

\section{Variables evaluadas}

Mortalidad de Adultos a los 5,10,15, 20 y 25 días Tiempo a la mortalidad del $50 \%$ de insectos (TL 50)

\section{Manejo del Experimento}

Con la finalidad de disponer de insectos para la prueba, se colocaron en lugares con alta incidencia de la plaga, (Cotopaxi y Chimborazo) trampas (sin insecticida alguno), de acuerdo a la metodología propuesta por Gallegos, et al. (3). Los insectos recolectados en el campo fueron llevados al laboratorio.

Los aislamientos de Beauveria sp. y $M$. anisopliae que se encuentran conservados en silicagel, se multiplicaron en cajas petri con medio PDA más harina de insecto de adulto de $P$. vorax .

Previamente a la prueba se realizó la potencialización de los aislamientos que sirvió para recobrar la virulencia del entomopatógeno en el hospedante en el cual se encontró. Los patógenos desarrollados en estos insectos se aislaron nuevamente en medio de cultivo PDA, más harina de adulto de $P$. vorax

La prueba de patogenicidad involucró el siguiente procedimiento. En primer lugar se procedió a colocar $10 \mathrm{ml}$ de agua destilada más dos gotas de tween 80 (emulsión) en las cajas petri que contenían esporas del hongo; y luego se realizó un raspado de este medio. En la suspensión de esporas, (5 x 106 en promedio) de cada aislamiento, se introdujeron por dos minutos 20 insectos adultos vivos de $P$. vorax (desinfectados previamente con cloretol al $0.05 \%$ y lavados con agua estéril) .Finalmente en cajas plásticas se colocó tierra estéril, hojas de papa y los insectos de la prueba; y se mantuvo para realizar la evaluaciones respectivas. 


\section{Resultados}

\section{Mortalidad de adultos de $P$. vorax}

Esta evaluación se realizó a los 5, 10, 15, 20 y 25 días después de la infección. El porcentaje de mortalidad se estableció relacionando el número de insectos muertos con el total de insectos con que se inició la prueba. El análisis de variancia mostró alta significación estadística para tratamientos en todos los días evaluados.

La mayor variación se presentó a los 5 días (CV 38.5\%), ésta se debió principalmente a que los aislamientos utilizados no actuaron en forma consistente sobre el insecto. Además debido a que los insectos de la prueba no tuvieron la misma edad, pudieron ofrecer diferente respuesta frente a los hongos. Esta variación disminuyó a medida que los tratamientos incrementaron su acción sobre los insectos, lo que se manifestó en la normalización de los resultados. Los coeficientes de variación de los demás días de evaluación se enmarcaron entre el 7 y $11 \%$.

La prueba de Tukey al 5\%, Tabla 1 indica que todos los aislamientos en estudio incrementaron su efectividad desde los 5 días, pero no en todos se alcanzó el $100 \%$ de mortalidad hasta los 25 días; lo que demuestra diferencia entre los aislamientos.

Tabla 1. Porcentaje de mortalidad de adultos de P. vorax a los 5, 10, 15, 20, y 25 días, en el estudio de la patogenicidad de aislamientos nativos de Beauveria sp. y Metarhizium anisopliae en Laboratorio, Santa Catalina Pichincha, 2002 (Prueba de Tukey al 5\%).

\begin{tabular}{|c|c|c|c|c|c|c|c|c|c|c|c|}
\hline \multirow{2}{*}{$\begin{array}{l}\text { Código } \\
\mathrm{t} 1\end{array}$} & \multirow{3}{*}{$\begin{array}{l}\text { Significado } \\
\text { San José de Minas } \\
\text { Santa Catalina }\end{array}$} & \multicolumn{2}{|c|}{$\begin{array}{c}\% \\
\text { mortalidad } \\
5 \text { días } \\
\end{array}$} & \multicolumn{2}{|c|}{$\begin{array}{c}\% \\
\text { mortalidad } \\
10 \text { días } \\
\end{array}$} & \multicolumn{2}{|c|}{$\begin{array}{c}\% \\
\text { mortalidad } \\
15 \text { días } \\
\end{array}$} & \multicolumn{2}{|c|}{$\begin{array}{c}\% \\
\text { mortalidad } \\
20 \text { días }\end{array}$} & \multicolumn{2}{|c|}{$\begin{array}{c}\% \\
\text { mortalidad } \\
25 \text { días }\end{array}$} \\
\hline & & 6.0 & $\mathrm{~cd}$ & 31.00 & cde & 69.00 & de & 76.00 & $\mathrm{~cd}$ & 82.00 & bcd \\
\hline \multirow{2}{*}{ t2 } & & 7.00 & bcd & 72.00 & $\mathrm{~b}$ & 96.00 & $a b$ & 96.00 & $a b$ & 96.00 & $a b$ \\
\hline & Chanchalo & 25.0 & a & 100.0 & a & 100.0 & a & 100.0 & a & 100.0 & a \\
\hline t3 & Sablog & 10.0 & bcd & 65.00 & $b$ & 78.00 & bcd & 90.00 & abe & 98.00 & a \\
\hline t4 & Huacona San José & 29.0 & $\mathrm{a}$ & 100.0 & a & 100.0 & a & 100.0 & a & 100.0 & a \\
\hline t5 & Santa Marta de & 5.0 & $\mathrm{~cd}$ & 27.00 & de & 57.00 & de & 80.00 & bcd & 90.00 & abcd \\
\hline t6 & Cuba & 4.0 & $\mathrm{~cd}$ & 32.00 & cde & 70.00 & cde & 86.00 & bcd & 93.00 & $a b c$ \\
\hline \multirow{2}{*}{ t7 } & FCA-CADET & 7.0 & bcd & 44.00 & c & 71.00 & cde & 80.00 & bcd & 89.00 & abcd \\
\hline & Guano & 4.0 & $\mathrm{~cd}$ & 100.0 & a & 100.0 & $\mathrm{a}$ & 100.0 & $\mathrm{a}$ & 100.0 & $\mathrm{a}$ \\
\hline t8 & San José de Huaca & 10.0 & bcd & 35.00 & $d$ & 61.00 & de & 77.00 & $\mathrm{~cd}$ & 80.00 & $\mathrm{~cd}$ \\
\hline t9 & Tumbaco & 0.0 & bcd & 38.00 & $\mathrm{~cd}$ & 66.00 & de & 81.00 & bcd & 86.00 & abcd \\
\hline $\mathrm{t} 10$ & Cabañal & 21.0 & $a b$ & 32.00 & cde & 75.00 & bcd & 84.00 & bcd & 87.00 & abcd \\
\hline $\mathrm{t} 11$ & Pull Chico & 8.0 & bcd & 46.00 & c & 63.00 & de & 76.00 & $\mathrm{~cd}$ & 86.00 & abcd \\
\hline
\end{tabular}




\begin{tabular}{|c|c|c|c|c|c|c|c|c|c|c|c|}
\hline+12 & Trebon & 0.0 & & 25.00 & de & 63.00 & de & 79.00 & bcd & 82.00 & bcd \\
\hline 112 & 4 esquinas & 1.0 & $d$ & 9.000 & $\mathrm{fg}$ & 31.00 & f & 50.00 & e & 50.00 & e \\
\hline 113 & compostera & 1.0 & d & 19.00 & ef & 51.00 & ef & 70.00 & d & 77.0 & d \\
\hline t14 & FCA- ESPOCH & 15.0 & $a b c$ & 93.00 & a & 100.0 & a & 100.0 & $\mathrm{a}$ & 100.0 & a \\
\hline t15 & Yacubamba & 1.0 & d & 28.00 & de & 91.00 & $a b c$ & 99.0 & a & 100 & a \\
\hline t16 & San francisco & 0.0 & d & 0.000 & g & & & 0.00 & $f$ & 0.000 & $f$ \\
\hline t17 & Testigo & & & & & 0.000 & g & & & & \\
\hline t18 & & & & & & & & & & & \\
\hline t0 & & & & & & & & & & & \\
\hline
\end{tabular}

Del análisis del porcentaje de mortalidad de adultos de $P$. vorax se determinó, a los 5 días, como los mejores tratamientos a t5, t3 y t12 con promedios de $29 \%, 25 \%$ y $21 \%$ respectivamente; mientras que a los 10 días los tratamientos t5, t3, t9 y 117 alcanzaron el 100\% de mortalidad.

Estos tratamientos se comportaron de forma muy similar a los 15 y 20 días. A los 25 días se pudo observar que hubo otro aislamiento que también alcanzó el $100 \%$ de mortalidad, este fue el t18. Los resultados obtenidos por los demás tratamientos fueron menores a los antes mencionados, lo cual demostró la presencia de aislamientos más eficientes que otros de acuerdo al lugar de origen.

Al realizar las comparaciones ortogonales se determinó que los aislamientos de Beauveria sp. ejercieron mayor control que los de $M$. anisopliae. Además el tratamiento testigo absoluto no produjo mortalidad durante toda la prueba, lo que determinó que los resultados obtenidos sean contundentes.

La Figura 1 muestra insectos adultos de $P$. vorax afectados por Beauveria sp. a los 10 días de infección. Este hongo inicialmente produce una masa blanca algodonosa para luego convertirse en pulverulenta sobre el insecto. La Figura 2 muestra insectos adultos de Premnotrypes vorax afectados por M.anisopliae. Este hongo inicialmente produce una masa blanca algodonosa para luego convertirse en verde oliva con apariencia pulverulenta.

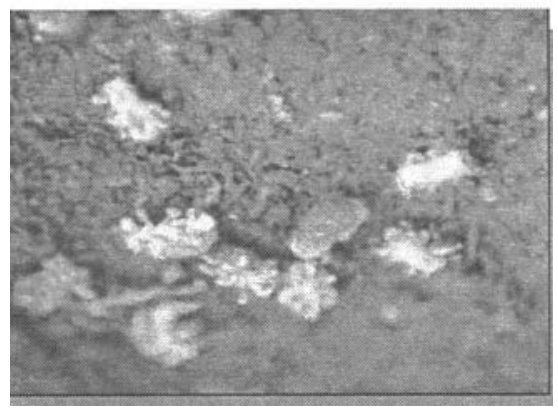

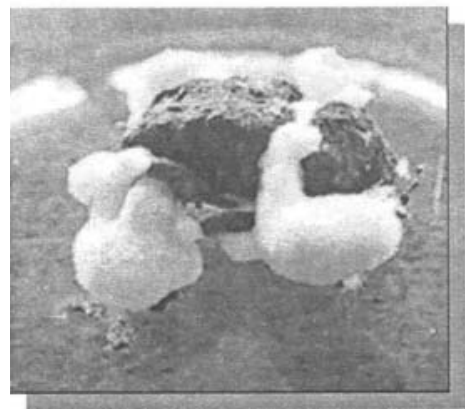

Figura 1. Adulto de P. vorax afectado por Beauveria sp.

Figura 2. Adulto de P. vorax afectado por $\mathrm{M}$, anisopliae 


\section{Tiempo letal medio}

Esta variable se analizó mediante una regresión lineal en la que se relacionó el porcentaje de mortalidad de cada tratamiento, durante las diferentes fechas de evaluación vs. los días de evaluación.

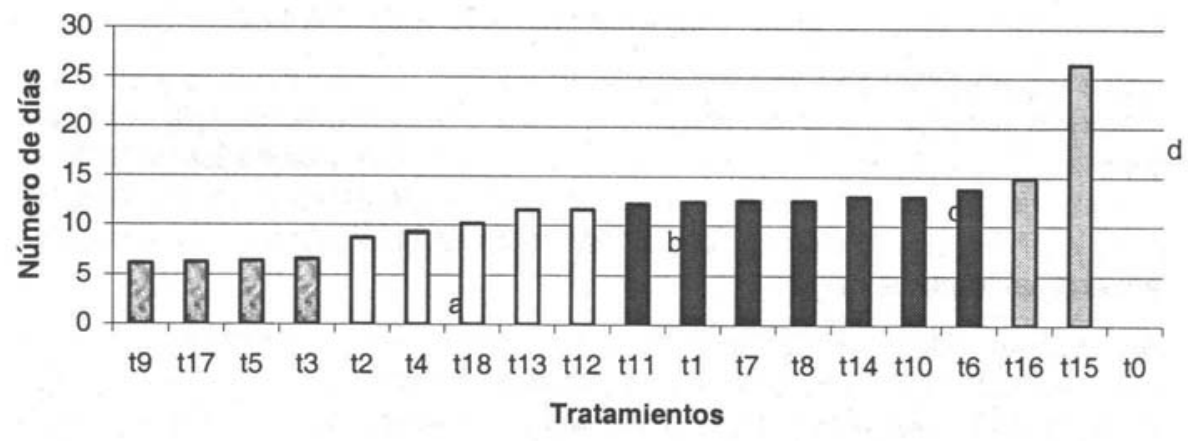

Figura 3. Tiempo Letal medio (TL50), en el estudio de la patogenicidad de aislamientos nativos de Beauveria sp y $\mathrm{M}$. anisopliae en Laboratorio, Santa Catalina, Pichincha 2002.

El cálculo del tiempo letal medio se realizó mediante la ecuación y=a+bx; la que permitió determinar el número de días que transcurrieron para obtener el $50 \%$ de mortalidad de los insectos de $P$. vorax sometidos a prueba.

$$
\begin{aligned}
& X=\text { Días de evaluación } \\
& Y=50 \% \text { mortalidad }
\end{aligned}
$$

En la Figura 3 se observan las diferencias alcanzadas por cada aislamiento en estudio; los mejores TL 50 fueron t3 Chanchalo (Cotopaxi) con 6.8 días y t5 Huacona San José (Chimborazo) con 5.8 días. El tratamiento testigo no presentó mortalidad de insectos de $P$. vorax durante el tiempo de evaluación. Los demás tratamientos requirieron de un tiempo de 10 días o más para eliminar el $50 \%$ de la población.

\section{Conclusiones}

- Los aislamientos nativos de los entomopatógenos de Beauveria sp. y $M$. anisopliae provenientes de las principales zonas productoras de papa fueron diferentes entre si en cuanto a patogenicidad para Premnotrypes vorax.

- De los aislamientos evaluados los de mayor patogenicidad para el control de $P$. vorax en laboratorio para las 2 variables fueron: t3 
(Chanchalo), t5 (Huacona S. J.), t9 (S. J. Huaca) y t17 (Yacubamba); el $100 \%$ de mortalidad de adultos de $P$. vorax alcanzaron a los 10 días de infección. El tiempo letal medio TL 50 fue de 6.6, 6.4, 6,6 y 6.3 días; Los aislamientos en los que el insecto presentó menor susceptibilidad fueron t2 (Santa Catalina), t7 (Santa Martha de Cuba), t15 (Compostera) y t16 (ESPOCH), El testigo no registro mortalidad.

\section{Recomendaciones}

- Para trabajos con entomopatógenos en primer lugar se debe proceder a formar un banco de cepas (aislamientos), para luego realizar evaluaciones que permitan encontrar diferencias de patogenicidad para un hospedante determinado

- Realizar pruebas en campo con las cepas mas eficientes identificadas en este estudio para el control de $P$. vorax

\section{Referencias Bibliográficas}

1. Alves, B. 1986. Controle microbiano de insetos. Primeira edicao. Editora Manole Itda. Sao Paulo, (Bra). p 246-247.

2. Crissman, C. 1999. Salud humana y cambios en las tecnologías de producción de papas en la eco-región alto andina del Ecuador (Proyecto Eco-Salud). Organización de las Naciones Unidas para la Agricultura y la Alimentación-Ecuador. Quito (Ec.). Boletín No. 10: 56.

3. Gallegos, P., G. Avalos, C. Castillo. 1997. El gusano blanco de la papa en el Ecuador Comportamiento y Control. Programa Nacional de Raíces y Tubérculos. D.N.P.V. INIAP Quito (Ecu). p. 22 - 25.

4. Gonzales, M., F. Posadas, P. Bustillo. 1993. Desarrollo de un Bioensayo para evaluar la patogenicidad de Beauveria bassiana sobre Hypothenemus hampei. Cenicafé 44(3); Caldas (Col);p.93-102

5. Torres H., A. Ortega, J. Alcazar, T. Ames, L. Palomino. 1993. Control Biológico del Gorgojo de los Andes (Premnotrypes spp.) con Beauveria brongniartii. Guía de investigación CIP 8, Centro Internacional de la papa, Lima, (Per), p.5-7-16. 